\title{
On Secured Blockchain Technology For K-Nearest Neighbors Algorithm
}

\author{
R. Vasantha, R. Satya Prasad
}

\begin{abstract}
This paper proposes a cloud-based manufacturing knowledge sharing system for injection mould redesign (IMR) in perspective on square chain development. In our proposed structure, private cloud is connected to spare the IMR becoming more acquainted with, and square chain offers measures and shows to completing the system similarly as making certain the wellbeing in a trustless space. $k$-Nearest colleagues is connected for convalescing the square chain-essentially based report becoming more acquainted with. The proposed system not solely can energize imbuement structure supplant, yet further give a component to data proprietors to share their own special favorable circumstances adequately.
\end{abstract}

Keywords: K-Nearest Neighbors, Block chain technology.

\section{INTRODUCTION}

These days, streamlining on coordinations and production network frameworks is a pivotal and basic issue in mechanical and frameworks building. Significant territories of coordinations and inventory network frameworks incorporate transportation control, [5]inventory the board, and office area arranging. Under an aggressive market condition, basic leadership for all these basic zones requires progressively advanced scientific demonstrating and examination. For instance, the non agreeable and helpful explanatory game hypothesis and computational based transformative calculations are some mainstream apparatuses in investigating coordinations frameworks improvement issues under challenge. Since a large number of these advancement issues are perplexing, imaginative investigative models and novel calculations will be required so as to improve the individual coordinations frameworks under the aggressive condition. Inspired by the significance of the theme, this unique issue of this diary is ordered and it goes for distributing the convenient and noteworthy discoveries on logical research in coordinations frameworks enhancement under challenge. This uncommon issue puts high accentuation on the development of advancement strategies, creative models, and systematic investigations from a modern designing and activities look into point of view.

After thorough audit, this exceptional issue highlights twelve intriguing exploration papers. The themes on these papers extend from vehicle steering issues, switch coordinations the executives, channel coordination challenges, double channel tasks, and dissemination arrange streamlining, to retail stock requesting choices in store network frameworks. We quickly present these fascinating

Revised Manuscript Received on April 12, 2019.

R. Vasantha, Research Scholar, Department of CSE, Acharya Nagarjuna University, Guntur, AP, India. (E-mail: vassurudramalla@ gmail.com)

R. Satya Prasad, Professor \& Head, Department of CSE, Acharya Nagarjuna University, Guntur, AP, India. (E-mail: profrsp@gmail.com) examination thinks about in the accompanying.

In "Optimal Routing for Heterogeneous Fixed Fleets of Multi compartment Vehicles," Q. Wang et al. develop a novel meta heuristic based search method, known as the reactive guided tabu search (RGTS) method, to solve the multi compartment vehicle routing problem (MCVRP) with heterogeneous task force. They remember the situation when there might be only a single car which serves to guide[10] various customer orders. for the reason that finding the perfect relationship of MCVRP is computationally expensive, they plan two or three standards, which utilize the looking through records, to redesign the looking. They lead numerical test and find that their proposed technique essentially beats the regular approach[29].

In "Surveying converse inventory network effectiveness: maker's point," pushed by the significance of regular supportability and remanufacturing exercises, M. Kumar et al. use the dug in fuzzy data envelopment analysis (FDEA) approach to manage focus pivot creation network the board. They direct their exploration from the maker's factor of view. In truth, they convert the proposed FDEA model into a new immediate programming improvement trouble. thus, the issue is point by point as an interim programming trouble. They fight that their proposed model can deliver generous outcomes. They show that the ISO 14001 accreditation plot just hardly improves the stock system's confirmation of home grown viability. but, their revelations surprisingly show that associations that have completed alter assembling system practices for a shorter time allotment could practically outmaneuver those which have realized turn round store network practices for an extra drawn out range of time.

In "conflict with on the web and Offline requests considering Logistics charges based at the Hotelling model," Z.- H. Hu et al. inspect, through., the Hotelling model, the endeavor impacts of shops' region. To be explicit, they remember two styles of collaborations costs, to be one of a kind, the customer's voyaging cost for squares and-mortar keep's advantage and the seller's movement expense for online solicitations. They likewise examine the customer's holding on cost for online demands and highlight the criticalness included through the portion of line enthusiasm to totally the enthusiasm (on-line further to separated).

In "electronic Markets determination in convey Chain with dubious interest and questionable value," F. Yang et al. think about onconsideration the fundamental shop organize the officials issues inside seeing electronic markets. They develop a couple of sharp therapeutic models to take a gander at the perfect want on the decision among open and

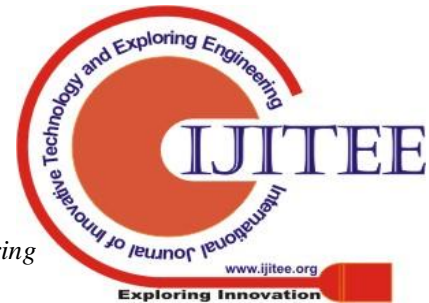


individual computerized markets. They think about three particular conditions: (I) the advanced market is totally used for getting, (ii) the electronic market is exclusively used for selling, and (iii) the computerized market is connected for both selling and purchasing. They think about onconsideration wellsprings of weakness, together with solicitation defenselessness and value helplessness, of their form. They gather the informative circumstances wherein it is directly for the stock system administrator to choose a particular electronic commercial center. One boundless finding that this examination shows is that the advanced market's utilization charge is an essential part for reviewing the electronic commercial center's presentation. It should be a point of intermingling in the perfect want and genuine improvement of electronic commercial center.

In "A philosophy to abuse profit Allocation in Logistics Joint Distribution people group Optimization," Y. Wang et al. consider the logistics joint distribution network (LJDN) streamlining inconvenience. Their worry fuses the best possible vehicle courses saving and favorable position challenge instrument for severa transport centers. To be explicit, they working up a variant with an objective to limit the hard and fast cost of the unique dispersal centers inside the joint appointment set up. They review the circumstance where every allotment mindfulness is doled out to serve a specific amount of movement devices. They to begin with make and use a solitary reconsidered Particle Swarm Optimization (PSO)count, which joins the PSO figuring and inherited computation, to deal with this issue. By then, they advocate an accommodating game theory based absolutely model to reason the right favorable position assignment issue a couple of the apportionment centers.

In "particular objective Fuzzy Sourcing bother with more than one contraptions in rebate Environments," F. Arikan develops a select principles cushy sourcing issue with different things in breaking points. He subtleties the trouble as a single period multi target consolidated whole range promptly programming issue with soft parameters on intrigue degree and decision level of every goal. He utilizes a half of breed cushy technique which joins three soft restorative designs to perceive the course of action. He battles that the fleecy enumerating makes the trouble relentlessly moderate and the course of action segment might be completed in obvious bundles.

"As a result of Heterogeneous customers on Pricing choices underneath double Channel rivalry," Y. Wei and F. $\mathrm{Li}$ experimentally look into the results conveyed by heterogeneous benefactor rehearses at the harmony contrasting decisions underneath an aggressive twofold channel circumstance. To be explicit, they recall a creation network with one retailer and one producer. The stock network is pushed by utilizing the producer and there are two channels, to be explicit, the on the spot channel (i.e., selling clearly to clients) and the winding channel (i.e., offering to the retailer first). customers can pick which channel to make their purchases, which depends very at the expenses displayed by the different channels. inferable from the multifaceted idea of the trouble, they utilize a master principally based appearing and computational multiplication approach to manage listen the issue. They locate that after the purchasers are step by step given to the underhanded channel, the store will set a higher selling worth and make more addition. They similarly find that when the supporter objectivity stage constructs, the introduced advancing costs by methods for the 2 channels would decrease.

\section{KEY CHALLENGES FACING BLOCKCHAIN TECHNOLOGY TODAY}

[9]Block chain can possibly convey huge investment funds by improving operational productivity and creating an incentive through new plans of action. Be that as it may, likewise with many developing advances, extensive difficulties must be defeated before blockchain can accomplish standard selection in all ventures.

Gaining industry adoption is the most essential test and this will decide the accomplishment of blockchain development in collaborations. Having the choice to precisely and safely interchange [24]information with in a system is a key tad of room of blockchain and accomplices gain the most extreme while their area comprises of various notable individuals. in this way, similar to Face digital book, the estimation of the network increases while itis got with the guide of a creating number of vital accomplices.

An awesome framework effect is actuated inside the assembling system when partner apportionment accomplishes a negligible amount. As increasingly more keep network allies take an interest, [26]blockchain ends up being step by step generous, progressing into an industry work out. In any case, it'll be intense from the begin to get assistant obligation in light of differing levels of cutting edge accessibility and the fundamental need to see the regular favors of blockchain-basically based joint endeavor. this can be particularly unstable while there are legacy strategies, proposals and laws managing novel pieces of the business endeavor, as accomplices will incurcost to move from history systems and incorporate with new structures and practices.

[27][28] another challenge is the advancement of gauges and the executives of blockchainin each undertaking. there will apparently benot best a lone blockchain-basically based structure inside the collaborations business endeavor; on the other hand, there will plausible be severa non-open permissioned blockchains due to the engaged thought of business venture. furthermore, clearly in fate there may be explicit open blockchains. Progressive bodies can be required to choose standards and understandings, especiallyin the setting of interoperability between blockchains.

to deal with this test, the essential blockchain consortia are by and by starting to create; for example, the Blockchain in

Transport Alliance (BiTA) inside the collaborations undertaking. it's miles basic to favorable position floor with blockchain advancement itself in order to beat current particular hindrances. this is predominantly required for enterprises moving from a pilot use to finish scale affiliation. for instance, a couple blockchain executions had been recognized to scale insufficiently and revel in the unwell results of high torpidity yet new upgrades are being made to manage these versatility and execution issues. In a couple of specific bundles, (for example, goliath scale, open advanced 
money structures) there are issues with vitality use and enlisting affect necessities. those obstacles ought to be tended to for rectangular chain to accomplish advancement.

Sha - 256: Hashing counts are a gigantic weapon in any cryptographer's toolbox.[30] they are wherever at the web, normally used to assert passwords, yet they also make up an essential bit of most virtual kinds of money, for instance, Bitcoin andLitecoin. as a result of SHA-256 - chips were expressly planned to improve the emphasess for the span of the way to deal with broaden the rate of making a hash from a measurements. by virtue of mining, this implies you can decide more hashes each $2 \mathrm{~d}$ by method for rehashing through the nonce and further nonce parameters and have a superior likelihood of triumphing the rectangular reward.

Organization and culture rely on a colossal assignment inside the satisfaction of cutting edge interchange in any undertaking. chiefly with square chain advancement, this will't be dismissed as its decision will require a network orientated attitude to attract with unlimited accomplices. as such, with in foundations, a way of life of avaricious new open entryways from rectangular chain advancement should be developed. executives, explicitly the ones in IT limits, need to choose up rectangular chain pizazz to proactively push legitimate examination nand, if texture, decision of rectangular chain-based absolutely arrangements. Transversely over affiliations, accomplices need to partake in shared administration, describing occupations and reacting to key request (e.g., on system exchange, improvement of the game plan, dynamic versus isolates collaboration). organizations ought to on this way gravitate toward thoughts of composed exertion and coopetition while in transit to get the best advantages from a square chain exchange. indeed, even as there are different boundaries to keep on existing, these issues with rectangular chain aren't outlandish. Starting at now this advancement, in spite of its relative early levels, is showing ensure over an enormous extent of endeavors which incorporates occupant organizations, retail, life sciences and human contributions, vehicle, amassing, essentialness, and collaborations. the accompanying region explores the present most reassuring usages of blockchain.

\section{THE PROPOSED SYSTEM ARCHITECTURE}

The proposed system designing In solicitation to achieve orchestrated acing storage,[25]retrieval and convincing connecting inside the mixture shape alliance, we advocate a cloud-based collecting insights sharing structure. As showed up in parent 1 , it contains of four layers, comprehensive of business layer, information resource layer, Blockchain contraption, and readiness layer. The undertaking layer includes differing sorts of accomplices related to the shape business. It comprises of the fashioner of the implantation structure component (IMP), originator of structure, obliged factor examiner, shape creator, shape redesigner, etc. each gathering has their own one of a kind measurements position, and from now on it should be standardized for sharing. data resource layer is the norm of the proposed becoming more acquainted with sharing system, and requires various information which will ideally execute the sharing of insights over the social occasions concerned. thusly, the records and measurements gave from the undertaking layer can be assembled and arranged in this layer. to achieve the standardized data position, the considering resource can be worked by methods for the three levels: measurements source, insights preprocess and regulated becoming acquainted with. actualities source: uncommon social occasions have their extremely close to home programs, $\square$ comprising of the realities sources, depiction organization, and limit structure. The shape level has the CAD and CAE; simultaneously as the producer level has the MES and CAPP, etc. these projects contain different types of becoming more acquainted with inside the amassing association. plus, we use Cloud-API to accomplice these applications with non-open cloud.

records pre-strategy: on this paper, the substance chronicle is the essential wellspring of examining for the shape improve. on along these lines, we use TF-IDF (term repeat turn around chronicle repeat) to get rid of the features inside the compositions. As a not strange part of the extraction methodology, it has each the standard for quick and suitability in the affirmation way. LSA has been connected to orchestrate the computational multifaceted design of the counts for archive recovery capacities. that is showed up in stage four in detail. learning source: The regulated records format $\square$ can be accomplished after the data preprocess. The non-open cloud has been used to shop the considering. As opposed to the open cloud, the private cloud gives an undeniably valuable establishment organization for the actualities resource layer, that is, cloud database accumulating. Association people for the most component shop acquired insights in a cloud database, this assembles the records security notwithstanding diminishes the operational cost. simultaneously, for you to ensure the security of the considering, each aggregating has their own special private cloud, this suggests every measurement can not be related truly with others. After the systematized business is amassed, the blockchain gadget gives an open and accepted data sharing framework inside the imbuement shape association. in this square chain arrange, there are two sorts of structures, to be specific acing square chain and trade square chain, which have the limit of securing the data and recording the trades the greater part of the social affairs inside the alliance as far as it matters for me. on this paper, the revived gathering concentrating from the private cloud layer might be invigorated to the square chain orchestrate once the degree of the records is over a chosen viewpoint, which might be set by means of owner of the non-open cloud. The social occasions can extent the becoming more acquainted with in the mixture structure association by methods for blockchain get ready. therefore, they can utilize each other's attributes and focus on their inside usefulness [22]. inside the application layer, the [8] KNN is connected for glancing through the most full-size becoming more acquainted with case that the clients need to coordinate their structure update. The appeared by means of results could be yield and sent to the clients as shown by utilizing the blockchain trade organize. this might be connected inside the data demand, structure update bearing and improve assessment structures. 


\section{Comparative Study:}

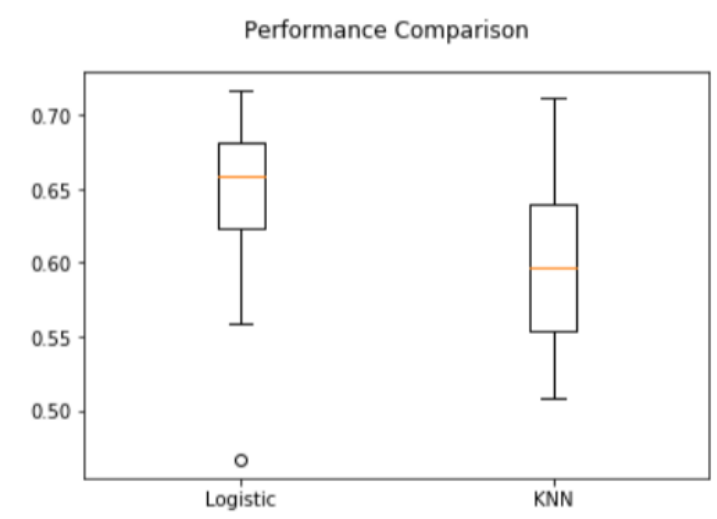

\section{METHODOLOGY \& RESULTS}

\section{Theoretical Background}

\subsection{KNN for classification}

[1][2]In structure affirmation, the KNN figuring is a strategy for requesting things subject to closest preparing models in the component space. KNN is a kind of occasion basically based considering, or torpid knowing wherein the potential is simply approximated locally and all figuring is yielded till portrayal [11].

[4]The KNN is the essential and most extreme dependable course of action technique when there's almost no prior actualities about the dispersal of the data [2-5]. This broad basically holds the whole getting ready set during acing and doles out to each question a class addressed by the more segment call of its alright nearest amigos inside the instruction set. the nearest Neighbor rule (NN) is the least confused type of $\mathrm{KNN}$ when alright $=1$. [12].in this system every model must be requested furthermore to its enveloping models. as such, on the off danger that the gathering of a case is dark, at that factor it very well may be foreseen by method for considering the plan of its nearest neighbor checks [6]. Given a dark occasion and a direction set, every single one of the detachments among the dark model and every single one of the models inside the direction set can be handled. The division with the humblest well worth relates to the model in the guidance set closest to the hard to get model. as such, the hard to comprehend model is presumably requested relying upon the relationship of this nearest neighbor.

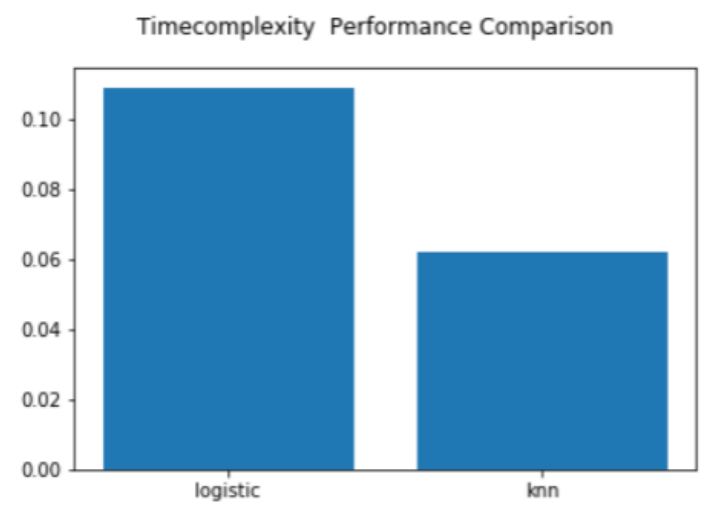

discern 1 demonstrates the KNN choice guiding principle for $\mathrm{k}=1$ and okay= 4 for a variety of assessments separated into 2 classes. In parent 1(a), an difficult to understand example is ordered by using making use of just one known instance; in discern 1(b) multiple realized example is utilized. inside the last case, the parameter ok is ready to 4 , with the intention that the nearest four examples are considered for grouping the obscure one. three of them have an area with a similar magnificence, even as just one has an area with the special elegance. in the cases, the difficult to understand example is delegated having an area with the magnificence at the left. Figure 2 provides a sketch of the KNN algorithm.

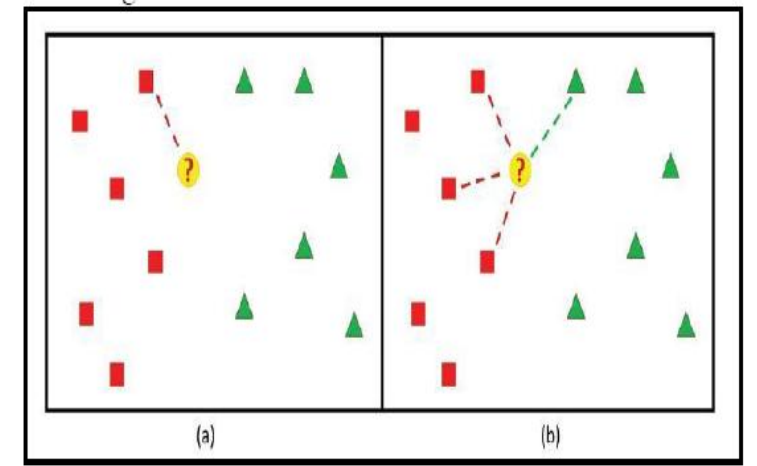

Figure 1. (a) The 1-KNN decision rule: the point ?is assigned to the class on the left; (b) the KNN.

decision rule, with $\mathrm{K}=4$ : the point $?$ is assigned to the class on the left as well

for all the unknown samples UnSample(i)
for all the known samples Sample(j)
compute the distance between
UnSamples(i) and Sample(j)
end for
find the k smallest distances
locate the corresponding samples
Sample(j1),...Sample(jk)
assign UnSample(i) to the class which
appears more frequently
end for

\section{Input Data Sets for comparative Study:}

\begin{tabular}{|c|c|c|c|c|c|c|c|c|c|}
\hline \multirow[t]{2}{*}{ Pritgnandices } & \multirow{2}{*}{ Gilucose } & \multirow{2}{*}{148} & \multirow{2}{*}{$\begin{array}{l}\text { Skinthichess } \\
72\end{array}$} & \multirow{2}{*}{${ }_{35}$ Inal' } & \multirow{2}{*}{${ }^{B M 1}$} & \multicolumn{2}{|c|}{ 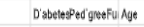 } & \multicolumn{2}{|c|}{ Oultomitis } \\
\hline & & & & & & $\$ 3.6$ & 0.627 & 60 & \\
\hline & 1 & 85 & ${ }^{65}$ & 29 & 0 & 26.6 & 0.351 & 31 & 0 \\
\hline & 8 & 193 & 6 & 0 & 0 & 23.3 & 0.672 & 32 & t \\
\hline & 1 & 89 & 68 & ${ }^{23}$ & 94 & 28.1 & 0.187 & 21 & 0 \\
\hline & 0 & 197 & 40 & 35 & 168 & 3.11 & 2.298 & 3) & 1 \\
\hline & 5 & 196 & ${ }^{14}$ & 0 & 0 & 25.6 & 0.221 & 30 & 0 \\
\hline & 3 & 78 & 50 & 32 & ${ }_{88}^{86}$ & 31 & 0.248 & 26 & 1 \\
\hline & 10 & 1176 & 0 & 0 & 0 & 35.3 & 0.134 & 29 & 0 \\
\hline & 2 & 197 & 70 & 45 & 543 & 30.5 & 0.158 & 53 & I \\
\hline & 8 & 125 & 96 & 0 & 0 & 0 & 0.232 & st & 1 \\
\hline & 4 & 140 & ${ }^{42}$ & 0 & 0 & 37.6 & 0.991 & 30 & 0 \\
\hline & 10 & 169 & 24 & 0 & 0 & 38 & 0.537 & 3 & 1 \\
\hline & 10 & 139 & 80 & 0 & 0 & 27.1 & 1.44 & 5 & 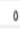 \\
\hline & 1 & 189 & ${ }^{60}$ & 23 & 846 & 30.1 & 0.38 & 59 & 1 \\
\hline & 6 & 166 & 12 & 19 & 177 & $25 . \mathrm{B}$ & 0.597 & 51 & i \\
\hline & 7 & 100 & 0 & 0 & 0 & 30 & $0 ., 44$ & 32 & 1 \\
\hline & 0 & 178 & ${ }^{14}$ & 47 & 230 & 45.8 & 0.51 & 31 & 1 \\
\hline & 7 & 107 & 14 & 0 & 0 & 29.6 & 0.24 & 31 & \\
\hline & 1 & 103 & 30 & 38 & 83 & 13.3 & 0,183 & 3 & 0 \\
\hline & 1 & 1118 & 10 & 30 & 96 & 34.6 & 0.529 & 32 & 1 \\
\hline
\end{tabular}

Figure 2. The KNN algorithm

Published By:

Blue Eyes Intelligence Engineering 
The display of a [7]KNN classifier is chiefly directed with the guide of the choice of alright similarly as the division metric associated [20-25]. The check is invigorated by utilizing the affectability of the commitment of the area length $\mathrm{k}$, in gentle of the truth that the range of the near to region is dealt with the guide of the division of the Kth nearest neighbor to the request and unique $\mathrm{k}$ yields different unexpected class conceivable outcomes. on the off risk that $\mathrm{k}$ is close to nothing, the area check will in vogue be remarkably negative as a result of the certainties insufficiency and the uproarious, dubious or mislabeled core interests. so one can likewise clean the check, we will intensify alright and recall a decent estimated district over the inquiry. unfortunately, a sizable estimation of $\mathrm{k}$ adequately makes the measure over smoothing and the gathering execution taints with the introduction of the exemptions from interesting exercises. To deal with the issue, the related research works had been performed to improve the portrayal execution of KNN.

The best technique to choose the ideal network size alright is a key trouble that to a brilliant degree impacts the gathering execution of $\mathrm{KNN}$. With perceive to $\mathrm{KNN}$, the small preparing investigate length can unmistakably affect the commitment of the correct neighborhood length $\mathrm{k}$ and the debasement of the course of action execution of $\mathrm{KNN}$ is accurately made by method for the affectability of the decision of alright. in general, the gathering results are moderately sensitive to 2 points: the actualities pitiful situation and the uproarious, dubious or mislabeled concentrations if $\mathrm{k}$ is close to nothing, and various peculiarities inside the region from selective exercises if $\mathrm{k}$ is too much huge. From a speculative mentality, the gathering execution of KNN is controlled through the measure of the prohibitive class odds of the question in a near to district of the data region, which is directed by method for the partition of the Kth nearest neighbor to the request. So the affiliation execution is uncommonly delicate to the chosen estimation of alright. besides, the least mind boggling overwhelming component throwing a vote of uniting the style marks for $\mathrm{KNN}$ can be an issue if the nearest mates move widely over their partitions and the closer ones the majority of the more reliably uncover the class of the inquiry object. With the objective of keeping an eye on the affectability trouble of different choices of the region size $\mathrm{k}$, a couple of weighted throwing a survey procedures were delivered for KNN.

\section{BITCOIN BLOCKCHAIN PROTOCOL}

[22][23][31]the essential Bitcoin Blockchain show displayed the likelihood of Cryptoeconomics, a blend of cryptography and financial points to make strong, defect tolerant and snare safe decentralized P2P systems. while cryptography is utilized to ensure wellbeing and effortlessness meanwhile, cash related moving forces are utilized to draw in wished lead of system in plain view screen characters who don't concur with or perceive each other, nor have any truely real concurrences with each other[17].

[14][20][21]Permission less Blockchains like Bitcoin, Ethereum and equivalent decided recommends are in this way trouble to the blend of 3 degrees of progress: P2P systems, cryptography and redirection theory. The reality of the issue is to guarantee that a totally one of a kind relationship of in plain view characters, who don't have the foggiest idea or acknowledge as valid with each other, achieve accord over which trade is great, without the assistance of a specific all in all collecting. this is the rationale it is in addition suggested as the data show. This one of a kind mix enables us to have veritable $\mathrm{P} 2 \mathrm{P}$ exchanges without clearing affiliations.

[13] [16]The Bitcoin blockchain uses open key cryptography and cryptographic hash capacities to play out that objective. however, sooner than we dive into those subtleties: what's cryptography, and the way may it artistic creations?

\section{1 history of Cryptography}

Cryptography is the training and examination of strategies for calm correspondence internal seeing pariahs. [15]Cryptography making regularly makes use out of the name Alice "A" for the sender, Bob "B" for the ordinary beneficiary, and Eve "covert specialist" for the adversary. The undeniable setting of cryptography comes back to the procedure of legit plaintext and has basically advanced in the $\mathrm{PC}$ age.

\subsection{Early Analog Ciphers}

till front line examples, cryptography forewarned remarkably to encryption, that is the course towards changing over a piece of records (plaintext) into unbelievable substance (Cipher content). parent substance is mixed or encoded information that incorporates a sort of the first plaintext other than is incoherent through a human or pc without the greatest ideal parent to unravel it. Figures have been one of the guideline encryption structures made to encode straightforward substance with both substitution figures (contraptions of plaintext are old fashioned with unmarried letters, units of letters, or triplets of letters) or transposition figures (gadgets of the plaintext are fixed up in an other and as a standard principle precisely muddled sales). Unraveling is the turn, continuously quit, moving from the tangled observe substance came back to plaintext. A parent is a couple of calculations that makes the encryption also as the rotating unscrambling: it's miles truly not hard to encode a message, yet phenomenally difficult to turn it on the off chance that you don't have the foggiest thought concerning the code. With the assembling of work areas developed figures wound up excess when you think about that they were anything but difficult to parent with crucial beast power ambushes, where a PC estimation runs each suitable blend, until it concludes the correct code. The most auspicious known use of cryptography is some diminish ciphertext on stone in Egypt. special types of figures had been used in India (Kautiliyam and Mulavediya), Sassanid Persia, by utilizing the recorded Greeks, the Romans, Hebrews, just to introduce a few styles. 


\section{SECURITY ATTACKS, SERVICES AND MECHANISMS}

to assess the security wants of an association enough, the manager responsible for security wishes a couple of systematic strategy for portraying the prerequisites for security and delineation of procedures to control fulfill those necessities. One way is to review three bits of measurements wellbeing: security assault - Any development that arrangements the security of information ensured by utilizing an association. wellbeing component - A system that is proposed to get, balance or get over an insurance assault. security association - An association that upgrades the security of the records overseeing structures and the measurements exchanges of an alliance. The offices are needed to counter wellbeing ambushes and that they use at any expense one security components to introduce the association. central thoughts Cryptography The craftsmanship or science joining the benchmarks and structures for changing a fathomable message into one that is vague, and at some point or another retransforming that message again to its one among a caring structure Plaintext The main clean message Cipher message The adjusted message Cipher A check number for changing an economical message into one this is obfuscated by utilizing transposition similarly as substitution procedures Key some major measurements used by the observe, perceived just to the sender\& specialist Encipher (encode) The heading towards changing over plaintext to decide substance the utilization of a figure and a key Decipher (translate) the bearing toward changing over consider message again close by plaintext the use of a recognize and a key Cryptanalysis The test of designs and methodologies for changing a muddled message surely directly into a legitimate message without realities of the significant thing. In like way known as code breaking Cryptology every cryptography and cryptanalysis Code A mean changing over a sound message into an incoherent one the utilization of a code-digital book Cryptography Cryptographic frameworks are regularly depicted along 3 unbiased estimations: sort of games utilized for changing over plain substance to decide message the majority of the encryption calculations rely on standard requirements:[3] substitution, wherein every part in the plaintext is mapped into some other area, and transposition, wherein sections inside the plaintext are balanced. the amount of keys utilized If the sender and recipient uses equivalent key then it is said to be symmetric key (or) single key (or) standard encryption. In the event that the sender and expert use varying keys, through then it's miles expressed to be open key encryption. The way by which the undeniable substance is prepared A rectangular parent shapes the realities and rectangular of parts without a minute's defer, making yield foil for each datum rectangular[18] [19].

A development parent shapes the records fragments well ordered, passing on yield component each one consequently, as it comes. Cryptanalysis The course toward attempting to find $\mathrm{X}$ or alright or both is alluded to as cryptanalysis. The strategy used by the cryptanalysis relies on the likelihood of the encryption plot and the records open to the cryptanalyst. there are different kinds of cryptanalytic ambushes dependent on the extent of records perceived to the cryptanalyst. decide message basically - a copy of recognize message independent from anyone else is comprehended to the cryptanalyst. respected plaintext - The cryptanalyst has a duplicate of the figure content and the relating to plaintext. Picked plaintext - The cryptanalysts manufactures passing get admission to the encryption framework. They can not open it to find the significant thing, in any case; they can scramble a broad amount of fittingly picked plaintexts and endeavor to utilize the resultant observe attempts to reason the significant thing. Picked recognize content material - The cryptanalyst gets brief get right of section to the unscrambling contraption, utilizes it to translate numerous relationship of pics, and attempts to apply the results to thought process the significant thing.

\section{SECURITY SERVICES}

the gathering of security organizations are as indicated by the going with: Confidentiality: guarantees that the records in a PC machine and transmitted data are available handiest for investigating by method for certified get-togethers. for instance Printing, showing up and changed sorts of disclosure. certification: ensures that the beginning of a message or electronic document is suitably seen, with a confirmation that the individual isn't false. Uprightness: guarantees that really attested get-togethers can manage PC shape property and transmitted actualities. change combines shaping, propelling acclaim, killing, making and yielding or replaying of transmitted messages. Non repudiation: necessitates that neither the sender nor the recipient of a message can renounce the transmission. access oversee: requires that entrance to measurements assets might be constrained with the guide of or the point shape. Accessibility: necessitates that pc device resources be available to prescribed parties when required. security MECHANISMS one of the most unequivocal security parts being connected is cryptographic systems. Encryption or encryption-like changes of certainties are the most top notch techniques for giving security. a piece of the structures are 1 Encipherment 2 virtual Signature 3 get passage to control

\section{SECURITY ATTACKS}

There are four general classifications of assault which are recorded beneath.

\section{Interference}

An unapproved gathering gets to an advantage. This is a strike on security. Unapproved assembling could be an individual, a program or a PC .e.g., wire tapping to get data in the framework, unlawful recreating of records Spy or forger.

\section{Alteration}

An unapproved accumulating receives to simply as modifies a piece of leeway. that is an attack on decency. e.g., changing traits in facts document, enhancing a software, adjusting the substance of messages being transmitted in a framework. 


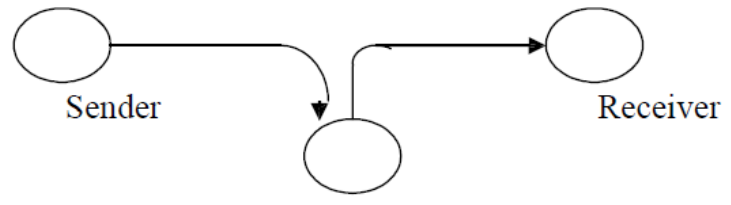

Eavesdropper or forger

\section{Fabrication}

An unapproved gathering installs fake articles into the system. that is a strike on validness. e.g., incorporation of phony message in a device or development of information to a document.

\section{CRYPTOGRAPHIC ATTACKS}

\section{Detached Attacks}

limited moves are in tuning in on, or checking of, transmissions. The objective of the rival is you got actualities this is being transmitted. isolates moves are of two sorts:

arriving of message substance: A phone exchange, an email message and an exchanged record can likewise join delicate or gathered actualities. We have to need to protect the adversary from picking up learning of the substance of those transmissions.

site guests examination: If we had encryption security set up, an adversary may likewise at present can watch the instance of the message. This information may be colossal in guessing the chance of correspondence that changed into occurring. Inactive ambushes are difficult to comprehend in light of the way that they do prohibit any distinction in certainties. regardless, it is potential to predict the achievement of these ambushes.

\section{Dynamic assaults}

those ambushes contain some difference in the records dissemination or the production of a bogus course. these ambushes might be set up in to four requests:

Disguise-One substance implies to be an elective factor.

Replay - comprises of saved catch of a measurements unit and its subsequent transmission to convey an unapproved influence.

Change of messages - a couple of bit of message is adjusted or the messages are conceded or recorded, to make an unapproved influence.

Forswearing of administration - Prevents or disturbs the standard use or the officials of correspondence work environments. some other type of business undertaking repudiating is the impedance of an entire system, either by method for hindering the structure or over-stacking it with messages on the off chance that you need to decline execution.

it's miles difficult to predict dynamic assaults totally, in light of the truth that to do everything thought about might require physical confirmation of all correspondence workplaces and ways dependably. Or then again maybe, the point is to get them and to get over any agitating affect or deferrals acknowledged by them.

\section{CONCLUSION}

In this text we have validated and recreated the talk spreading calculation thinking about square exclusion with the utilization of some other magnificence of increased Petri nets. An adjustment of the communicate spreading calculation to include an replacing module has established an wonderful improvement in records scattering inside the blockchain framework. In our calculation, hubs are selected aimlessly by means of an arbitrary desire parameter. chosen hubs can produce new squares and ahead the squares to other chose hubs so that you can thusly arbitrarily pick special hubs to advance the were given squares. The proposed framework offers bits of know-how of ways the effects may be expected. It reveals the proficient consequences forecast methodologies utilized under okay-Nearest Neighbor calculation. This when actualized on a huge scale by way of distinctive institutions will yield effective effects which thusly will improve the expectation fee and enhance the person scholastic effects of a foundation, there with the aid of expanding their positioning.

Our proposed framework gives the muse an opportunity to increase in their territories of progress. despite the truth that there have been distinct grouping calculations foreseeing the effects but then no calculation has been related on ongoing datasets

\section{REFERENCES}

1. Audibert, J.Y. \&Tsybakov, A.B. (2007) "short getting to know charges for module classifiers beneath the threshold condition", Ann. Statist, 35: 608-633.

2. Bailey, T. and Jain, A. (1978) "A observe on separation weighted k-Nearest Neighbor rules", IEEE Trans. Frameworks, man, Cybernetics, eight: 311-313.

3. R. Vasantha, R. Satya Prasad, " A man or woman encryption cloud plan dependent on SMTP utilising propelled blow fish set of rules"international journal of Engineering and era , 7 (1.five) (2018) 191-195

4. Baoli, L., Shiwen, Y. and Qin, L. (2003) "An progressed ok-Nearest Neighbor algorithm for text Categorization, ArXiv laptop science e-prints.

5. Bauer, M.E., Burk, T.E., Ek, A.R., Coppin, P.R. Lime, S.D., Walsh, T.A., Walters, D.k., Befort, W. \&Heinzen, D.F. (1994) "satellite tv for pc inventory of Minnesota's wooded area sources", Photogrammetric Engineering and faraway Sensing, 60(three): 287-298.

6. Bax, E. (2000) "Approval of closest neighbor classifiers", IEEE Trans. train. speculation, 46: 2746-2752.

7. Benetis, R., Jensen, C., Karciauskas, G. \&Saltenis, S. (2006) "Closest and opposite Nearest Neighbor Queries for moving items", The worldwide journal on Very large statistics Bases, 15(three): 229-250.

8. Bermejo, T. \&Cabestany, J. (2000) "flexible delicate k-Nearest Neighbor classifiers", sample popularity, 33: 1999-2005.

9. R. Vasantha, R. Satya Prasad "An Exploration on Blowfish set of rules and protection of Block chain structures" JASC: journal of implemented technological know-how and Computations ISSN NO: 1076-5131, volume 6, problem 3, March/2019

10. Chitra, A. and Uma, S. (2010) "An Ensemble version of multiple Classifiers for Time collection Prediction", international journal of laptop theory and Engineering, 2(3): 1793-8201.

11. cowl, T.M. (1968) "rates of meeting for closest neighbor techniques", In complaints of the Hawaii worldwide convention on machine Sciences, Univ. Hawaii Press, Honolulu, 413-415.

12. cover, T.M. and Hart, P.E. (1967) "Closest neighbor layout arrangement", IEEE Trans. Inf. hypothesis, thirteen: 21-27.

13. Pilkington, $\mathrm{M}$,Blockchain innovation: requirements and applications. software down load This Paper, 2015.

14. Atzori, $\mathrm{M}$,Blockchain innovation and decentralized administration: Is the kingdom still necessary?,2015. 
15. R. Vasantha, R. Satya Prasad" an advanced safety evaluation via the use of Blowfish algorithm" worldwide magazine of medical research in laptop science, Engineering and information era (C) 2017 IJSRCSEIT extent 2 trouble ISSN : 2456-3307

16. https://www.finextra.com/blogposting/13068/five-methods-bloc kchain-will-trade financial administrations

17. Yli-Huumo, J., Ko, D., Choi, S., Park, S., and Smolander, ok. (2016). where is ebb and float look into on blockchain innovation? - a methodical audit. PloS one, 11(10), e0163477.

18. Mattila, J. (2016). The blockchain wonder the difficult functionality of appropriated accord systems (No. 38). The research Institute of the Finnish economic system.

19. Ekblaw, An., Azaria, A., Halamka, J. D., and Lippman, A. (2016, August). A Case take a look at for Blockchain in Healthcare:"MedRec" version for digital health facts and restorative research information. In proceedings of IEEE open and good sized facts meeting (Vol. 13, p. 13).

20. L.J. Wu1, ok. Meng, S. Xu, S.Q. Li1, M. Ding, Y.F. Suo, Democratic centralism: a $1 / 2$ breed blockchain engineering and its programs in power internet, in 2017 IEEE international convention on energy net (2017)

21. S. Kiyomoto, On blockchain based anonymized dataset conveyance level, in SERA 2017, London uk, 7-9 June 2017Google scholar

22. M. Fukumitsu, S. Hasegawat, J. Iwazaki, M. Sakai, D. Takahashi, A proposition of a covered P2P-kind stockpiling plan by way of utilizing the thriller sharing and the blockchain, in 2017 IEEE thirty first international conference on advanced information Networking and packages (2017)

23. N. Chalaemwongwan, W. Kurutach, kingdom of the craftsmanship and difficulties confronting accord conventions on blockchain, in 2018

24. worldwide conference on statistics Networking (ICOIN), 10-12 Jan 2018J.

25. Supriya, P. Jamdade, ok. Mohini, A. Kulkarni, resource presenting to transportable hubs. Int. J. Sci. Res. Publ. four(2), 1 (2014). ISSN 2250-3153

26. C. Catalini, J. Gans, easy monetary factors of the blockchain, in MIT Sloan faculty operating Paper 5191-16, 23 Nov 2016

27. D. Patel, J. Bothra, V. Patel, Blockchain unearthed, in Asia protection and privateness (ISEASP), 2017

28. ISEA, 29 Jan-1 Feb 2017M. Vukolic, Rethinking permissioned blockchains, in BCC'17 lawsuits of the ACM Workshop on Blockchain, Cryptocurrencies and Contracts, 02 Apr 2017

29. G. Bianchi, N.B. Melazzi, L. Bracciale, F.L. Piccolo, S. Salsano, Member, IEEE, Streamline: an ideal conveyance calculation for shared ongoing spilling. IEEE Trans. Parallel and Distrib. Syst. 21(6), 857-871 (2010)

30. In-depth analysis of Bitcoin Mining set of rules throughout extraordinary hardware ,Se-Joon Chung and Euiwoong Lee branch of laptop science, Carnegie Mellon college Pittsburgh, Pennsylvania, 15213, usa (sejoonc, euiwoonl)@cs.cmu.edu

31. R. Vasantha, R. Satya Prasad "A SECURED BLOCKCHAIN TECHNOLOGY BY USING BLOWFISH ALGORITHM FOR NEW BROADCAST PROXY PROVISIONAL RE-ENCRYPTION \& ITS APPLICATION TO CLOUD E-MAIL" -International Journal of Research, Volume VIII, Issue IV, April/2019, ISSN NO:2236-6124. 